\title{
PIDD orchestrates translesion DNA synthesis in response to UV irradiation
}

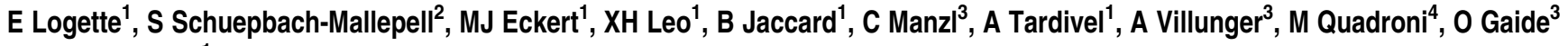 \\ and J Tschopp ${ }^{*, 1}$
}

PIDD has been implicated in survival and apoptotic pathways in response to DNA damage, and a role for PIDD was recently identified in non-homologous end-joining (NHEJ) repair induced by $\gamma$-irradiation. Here, we present an interaction of PIDD with PCNA, first identified in a proteomics screen. PCNA has essential functions in DNA replication and repair following UV irradiation. Translesion synthesis (TLS) is a process that prevents UV irradiation-induced replication blockage and is characterized by PCNA monoubiquitination and interaction with the TLS polymerase eta (pol $\eta$ ). Both of these processes are inhibited by p21. We report that PIDD modulates p21-PCNA dissociation, and promotes PCNA monoubiquitination and interaction with pol $\eta$ in response to UV irradiation. Furthermore, PIDD deficiency leads to a defect in TLS that is associated, both in vitro and in vivo, with cellular sensitization to UV-induced apoptosis. Thus, PIDD performs key functions upon UV irradiation, including TLS, NHEJ, NF- $\kappa$ B activation and cell death.

Cell Death and Differentiation (2011) 18, 1036-1045; doi:10.1038/cdd.2011.19; published online 18 March 2011

DNA lesions can result from endogenous metabolic processes as well as from exogenous DNA damaging agents. In both cases, different cellular responses are engaged (cell cycle arrest, repair pathways or apoptosis) to maintain genetic integrity. PCNA (proliferating cell nuclear antigen) acts as a DNA sliding clamp, which helps loading of replicative DNA polymerases. PCNA is involved in several forms of DNA repair, such as NER (nucleotide excision repair), BER (base excision repair) and MMR (mismatch repair), and also in other aspects of DNA metabolism, such as replication, chromatin assembly and cohesion. ${ }^{1}$ PCNA mediates these different functions through interactions with proteins specific to each process. $^{2}$ Many of these PCNA interacting proteins (PIPS) contain a so-called PIP-box. ${ }^{1,3}$ Competition between different partners for the same binding site on PCNA is one of the mechanisms that coordinate the functions of PCNA in DNA replication and repair. PCNA is loaded around the DNA by the conserved chaperone-like clamp loader complex RFC (replication factor $\mathrm{C}$ ), consisting of five subunits (RFC1 to RFC5). ${ }^{4,5}$ The RFC subunits all contain a PIP-box and thereby physically interact with PCNA. ${ }^{6}$ Once PCNA is linked to the DNA, the RFC complex is ejected from the clamp to allow DNA polymerase access to the clamp. ${ }^{7}$

The protein with the strongest affinity for PCNA is the PIPbox containing p21 (Cip1/Waf1), ${ }^{8}$ a potent cyclin-dependent kinase (CDK) inhibitor involved in cell cycle regulation, except in response to UV, where cell cycle arrest is independent of p21. ${ }^{9}$ P21 binds to PCNA and inhibits its activity, ${ }^{10,11}$ mainly because p21 obstructs the interaction of PCNA with DNA replication and repair factors. ${ }^{12-15}$ In vitro studies have shown that p21 also inhibits the loading of PCNA onto DNA, ${ }^{7}$ thereby compromising DNA repair. ${ }^{9} \mathrm{UV}$ is one of the major exogenous sources of DNA damage. The most common photoproducts induced by UV irradiation are CPDs (cyclobutane pyrimidine dimers). As a consequence, replicative DNA polymerases are halted at DNA lesions, resulting in replication arrest. To avoid this, two principal pathways are engaged that both require PCNA: NER, the main repair pathway by which bases damaged by UV irradiation are replaced using the high-fidelity polymerases $\delta$ and $\varepsilon$ and TLS (translesion DNA synthesis), a lesion bypass process that has evolved to avoid excessive fatal arrest of replication during $S$ phase. ${ }^{16}$ TLS is a fundamental mechanism for tolerating DNA damage that has escaped repair, carried out by specialized low stringency TLS polymerases (the Y-family and B-family, for review see ref. 17), which synthesize across a wide variety of DNA lesions. Particularly in response to UV irradiation, polymerase eta $(p o l \eta)$ has the unique property of incorporating nucleotides opposite CPDs with similar efficiency than undamaged DNA. ${ }^{18,19}$ In mammalian cells, the major modification of $P C N A$ in response to UV is monoubiquitination at lysine- $164^{20}$ mediated by the Rad6-Rad18 complex. ${ }^{21,22}$ This monoubiquitination alters the affinity of PCNA for different polymerases, such as increasing the affinity for pol $\eta$, that is then loaded onto the DNA and performs TLS past the UV lesions. ${ }^{23,24}$ Even though the effect of p21 on PCNA function

\footnotetext{
${ }^{1}$ Department of Biochemistry, University of Lausanne, Epalinges, Switzerland; ${ }^{2}$ Department of Dermatology-Immunology, Geneva Medical Center, rue Michel Servet 1 , Geneva, Switzerland; ${ }^{3}$ Division of Developmental Immunlogy, Biocenter, Innsbruck Medical University, Innsbruck, Austria and ${ }^{4}$ Protein Analysis Facility, Center for Integrative Genomics, University of Lausanne, Epalinges, Switzerland

*Corresponding author: J Tschopp, Department of Biochemistry, University of Lausanne, Ch. des Boveresses 155, CH-1066 Epalinges, Switzerland.

Tel: + 4121692 5738; Fax: + 4121692 5705; E-mail: jurg.tschopp@ unil.ch

Keywords: PCNA; PIDD; TLS; UV

Abbreviations: TLS, translesion synthesis; NHEJ, non-homologous end joining; PIDD, p53-induced protein with a death domain; CPDs, cyclobutane pyrimidine dimers Received 04.10.10; revised 27.1.11; accepted 28.1.11; Edited by B Zhivotovsky; published online 18.3.11
} 
is still controversial, several reports describe p21 as an inhibitor of PCNA monoubiquitination and thus TLS is induced by UV irradiation. ${ }^{9,25-27}$

PIDD (p53-induced protein with a death domain) was initially described as a p53-inducible gene. ${ }^{28}$ PIDD contains $N$-terminal Leucine Rich Repeats (LRR), two intermediate ZU5 domains and a $C$-terminal Death Domain (DD). ${ }^{29}$ PIDD can act as a molecular switch able to activate either a prosurvival or a pro-death program in response to DNA damage depending on its auto-proteolytic processing and the composition of proteins incorporated into the so-called PIDDosome. ${ }^{30}$ By recruiting RIP-1 and Nemo, PIDD activates the pro-survival NF- $\kappa$ B pathway, ${ }^{31}$ whereas recruitment of RAIDD and caspase-2 to the complex sensitizes cells to apoptosis. ${ }^{29}$ Recently, PIDD association with DNA-PKcs and caspase-2 was also shown to act on the NHEJ (non-homologous endjoining) repair pathway in response to $\gamma$-irradiation, ${ }^{32}$ suggesting a role for PIDD and caspase-2 in DNA repair. Here, we identify PIDD as a new interaction partner of PCNA. We provide evidence that PIDD modulates the p21-PCNA interaction and find that in response to UV-C, PIDD deficiency leads to a defect in PCNA monoubiquitination and a decrease of pol $\eta$ recruitment, whereas overexpression of PIDD has the opposite effects.

\section{Results}

PIDD forms a complex with PCNA and RFCs. To identify proteins that physically interact with PIDD, we immunoprecipitated Flag-tagged PIDD from stably transfected 293T cells, and analyzed PIDD-associated proteins by proteomics (Table 1). A substantial number of proteins were found that are known to have a role in DNA replication and/or repair. Most notably, almost all members of the 'PCNA-RFC-pol $\delta$ ' complex were detected. We, thus, decided to investigate a possible role of PIDD in DNA replication and repair.

Table 1 Functional categorization of PIDD-associated proteins identified by proteomics analysis as described in supplemental experimental procedures

\begin{tabular}{|c|c|}
\hline Category & Representative proteins \\
\hline PIDD & RAIDD/caspase-2 \\
\hline Heat shock/chaperones & - \\
\hline Replication factor & 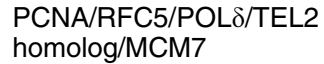 \\
\hline RNA polymerase & RPB2/RPAP3 \\
\hline Elongator complex & PP6 \\
\hline DNA damage/repair & $\begin{array}{l}\text { DDB1/FANCl/DNA-PKcs/ } \\
\text { XPD/XPG }\end{array}$ \\
\hline Histone modification & ANP32B \\
\hline Apoptosis & HAX1/AIF \\
\hline Cell cycle & - \\
\hline Ubiquitination & TRIM32 \\
\hline Sumoylation & SENP3 \\
\hline $\begin{array}{l}\text { Nuclear organization/ } \\
\text { nucleoporins }\end{array}$ & NUP93 \\
\hline Regulation of REDOX state & PRDX1 \\
\hline Transcription & - \\
\hline
\end{tabular}

Only proteins found in all experiments are listed. Data are representative of three independent experiments
In co-immunoprecipitation experiments with overexpressed PIDD, we could confirm that PIDD interacts with PCNA, RFC5 (Figure 1a) and RFC4 (Supplementary Figure S1a) as well as with the corresponding endogenous proteins (Figure $1 \mathrm{~b}$, lane 2). This interaction was specific as for example, the DDcontaining FADD protein showed no affinity for PIDD (Supplementary Figure S1b) and knockdown of PCNA and RFC5 resulted in the disappearance of the respective proteins in the PIDD complex (Figure 1b, lanes 3 and 4). This knockdown experiment also shows that the strong interaction of PIDD with PCNA was not dependent on the presence of RFC5, and neither was the PIDD-RFC5 interaction dependent on PCNA. The reconstitution of the PIDD-PCNA-RFC5 complex by overexpression appeared to be independent of the DNA damage response, as UV-C treatment did not alter the composition of the complex (Figure 1a) (however, this UV independency observed with overexpressed proteins is in contrast with the results obtained with endogenous proteins, see below).

PIDD is constitutively auto-processed giving rise to a 48-kDa $N$-terminal fragment containing the LRR (PIDD-N) and a $51-\mathrm{kDa} C$-terminal fragment (PIDD-C) containing the death domain (DD). The latter undergoes further cleavage resulting in a $37-\mathrm{kDa}$ fragment (PIDD-CC) (Figure 1d). Whereas initially formed PIDD-C mediates the activation of $\mathrm{NF}-\kappa \mathrm{B}$, subsequent formation of PIDD-CC causes caspase- 2 activation. $^{30}$

PIDD, through its DD, was previously shown to interact with RAIDD, RIP1 and DNA-PKcs. ${ }^{29,31,32}$ We therefore investigated whether this domain was also implicated in the interaction with PCNA. Using different non-cleavable mutants of PIDD previously described, ${ }^{30}$ we found that only the fulllength protein and the PIDD-C fragment interacted with PCNA (Figure 1c). Taken together, these results indicate that PIDD forms a complex with PCNA and RFC5, which does not rely on the DD and therefore is distinct from the caspase-2-activating PIDDosome. More in-depth mapping experiments indicated that the Zu5 domains of PIDD are required for interaction with PCNA and RFC5 (Figure 1e). Deletion mutant of PCNA indicate that the C-terminal part of PCNA is important for interaction with PIDD and especially the Zu5 domains (Figure 1f).

PIDD-PCNA interaction occurs transiently in the nucleus and is UV-C dependent. After a $1 \mathrm{~h}$ treatment of cells with $100 \mathrm{~J} / \mathrm{m}^{2} \mathrm{UV}-\mathrm{C}$, there appeared to be no change in complex formation of overexpressed PIDD and PCNA protein (as shown above in Figure 1a and Supplementary Figure S2a). As this was a surprising and unexpected observation (probably because of the overexpression system used), we investigated a possible effect of UV-C treatment on PIDDPCNA interaction in a more physiological setting and found that PIDD interaction with endogenous PCNA was indeed UV-C dependent $\left(50 \mathrm{~J} / \mathrm{m}^{2}\right)$. A transient increase in the interaction occurring $30 \mathrm{~min}$ after irradiation was observed in the nucleus (Figure 2a). Most interestingly, the kinetics of the nuclear complex formation is in agreement with previous results showing a peak of PCNA-based repair complex formation $30 \mathrm{~min}$ after UV irradiation. ${ }^{33,34}$ It is worth noting that the PIDD-CC fragment is absent in the nuclear 


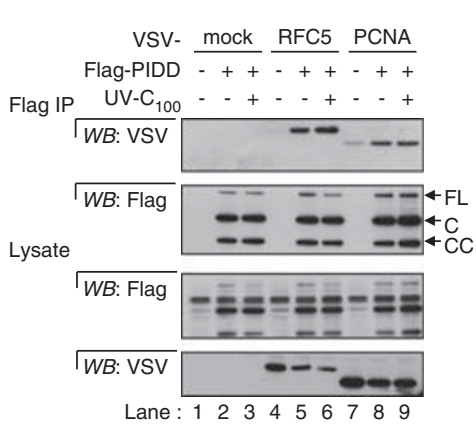

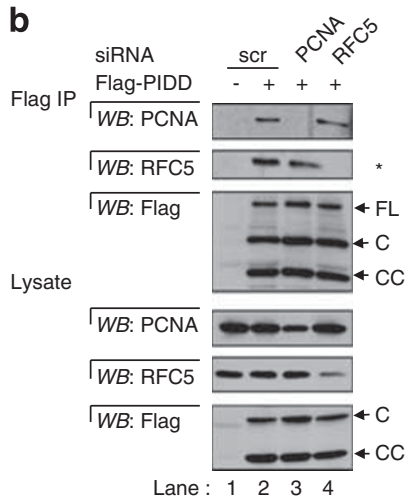

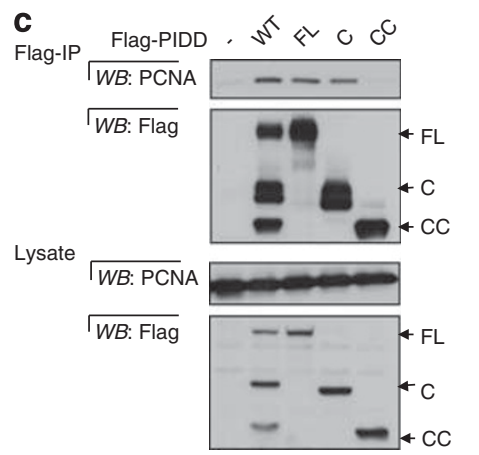

e

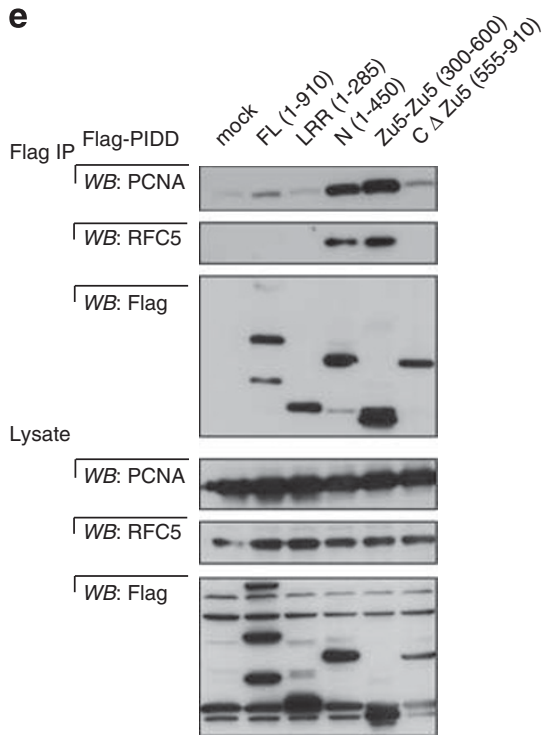

d

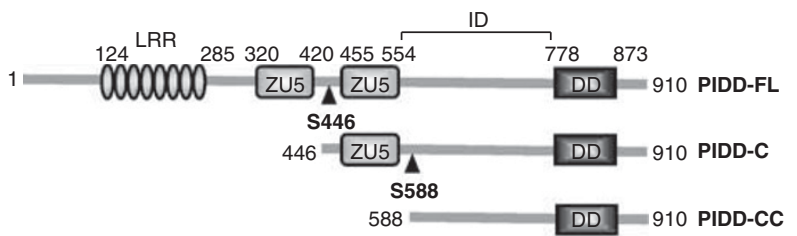

$1=0000000 \quad$ ZU5 $445 \quad$ PIDD-N

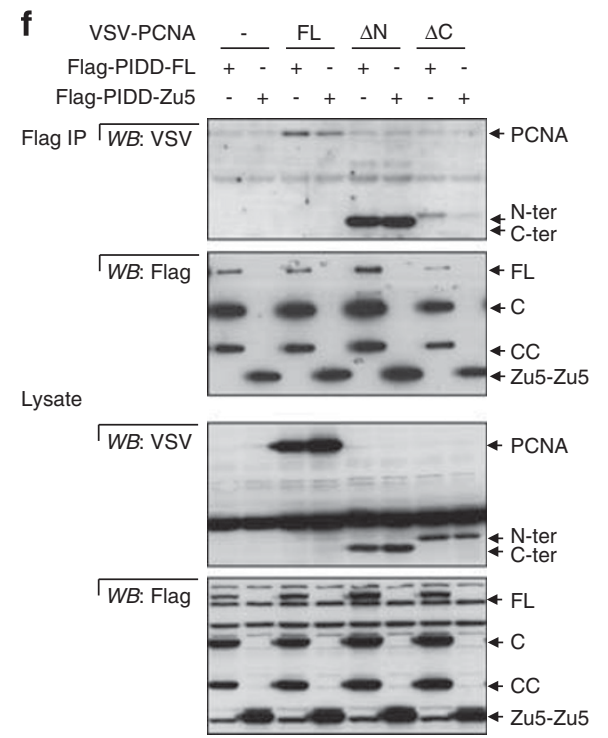

Figure 1 PIDD interacts with PCNA and RFC5 through its N-terminus. (a) PIDD interacts with overexpressed PCNA and RFC5: different VSV-tagged constructs were cotransfected in 293T cells with Flag-tagged PIDD and tested for interaction by co-immunoprecipitation (after $100 \mathrm{~J} / \mathrm{m}^{2} \mathrm{UV}$-C irradiation where indicated). See also Figures S1a and S1b. (b) PIDD interacts with endogenous PCNA and RFC5: scramble or specific siRNAs were cotransfected with Flag-tagged PIDD or a control vector in 293T cells and interactions with endogenous RFC5 and PCNA were analysed by anti-Flag IP $\left({ }^{*}=\right.$ a long exposure was required to detect endogenous RFC5). (c) PIDD-CC fragment does not interact with PCNA: 293T cells were transfected with wt or different non-cleavable mutants of PIDD, and endogenous PCNA interactions were analyzed by anti-Flag IP. (d) Structure and processing of PIDD: amino acids positions of each domain are indicated (LRR, Leucine Rich Repeat; ID, Intermediate Domain; DD, Death Domain). (e) Different fragments of PIDD were overexpressed in 293T cells and interactions with endogenous RFC5 and PCNA were analysed by anti-Flag IP. (f) Deletion constructs of PCNA lacking or the $\mathrm{C}$-ter $(\Delta \mathrm{C}=\mathrm{PCNA}$ aa1-120) or the Nter regions $(\Delta \mathrm{N}=\mathrm{PCNA}$ aa130-261), were cotransfected with Flag-tagged PIDD-FL or Zu5-Zu5 in 293T cells and tested for interaction by co-immunoprecipitation. (a-f) All the experiments were repeated at least two times. FL, PIDD Full-length; C, PIDD-C fragment; CC, PIDD-CC fragment as described in panel $\mathrm{d}$ (this nomenclature is conserved in all the figures)

immunoprecipitation, the major forms observed being the full-length protein and the C-fragment, confirming the mapping experiments that showed no interaction between
PCNA and PIDD-CC. The results suggest that the full-length and/or the PIDD-C fragment are important for the PCNA interaction. Similar kinetics and localization of PIDD-PCNA 
a
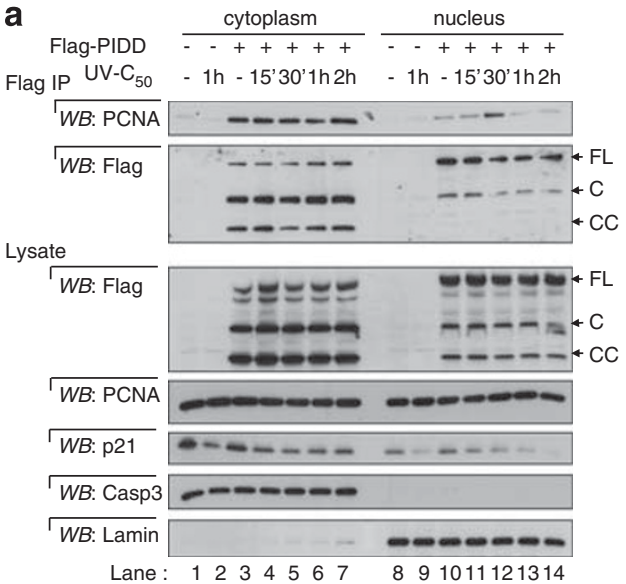

b

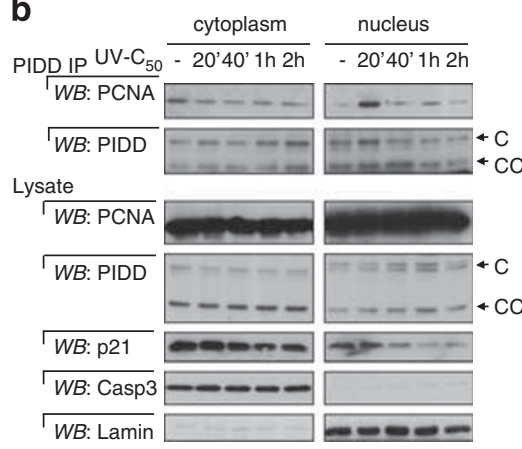

C

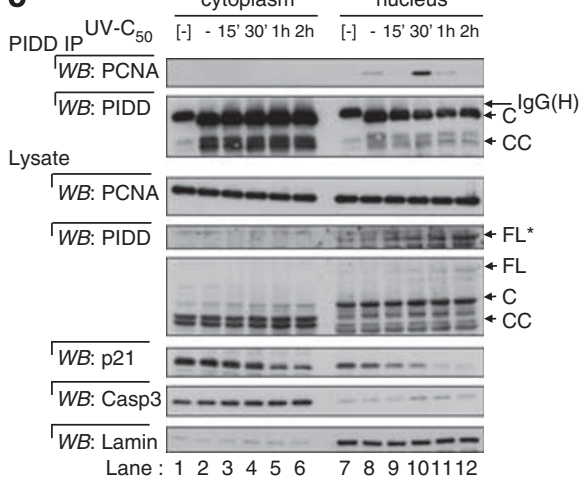

Figure 2 PIDD-PCNA interaction is regulated in nucleus by UV-C irradiation. (a) PIDD-PCNA interaction increases in nucleus after UV-C irradiation: 293T cells were transfected with Flag-tagged PIDD, subjected to cytoplasm-nuclear fractionation as described in 'experimental procedures', and endogenous PCNA interaction was analyzed in both fractions by anti-Flag IP after different times of $50 \mathrm{~J} / \mathrm{m}^{2}$ UV-C irradiation. (b) Endogenous PIDD and PCNA interact in nucleus in response to UV-C irradiation: the same experiment as in figure (a) except that endogenous PIDD was immunoprecipitated. See also Supplementary Figure S2b. (c) Endogenous PIDD and PCNA interact in nucleus in a keratinocytes cell line: the same experiment as in figure (b) was performed in $\mathrm{HaCaT}$ cells. $\left([-]=\mathrm{IP}\right.$ control was done with a control lgG antibody, ${ }^{*}=a$ long exposure was required to detect PIDD-FL). (b and $\mathbf{c})$ The hardly detectable endogenous PIDD-FL is only detectable with really high exposure (FL*). (a-c) The p21 degradation was analysed in parallel in total extracts. Caspase-3 and lamin were used as control of cytoplasmic and nuclear extracts, respectively. Data are representative of at least three repetitions

interaction were also found when exclusively endogenous proteins were analyzed in $293 \mathrm{~T}$ cells, in both total extracts (Supplementary Figure S2B) and nuclear fractions (Figure 2b). To strengthen these data, we also tested the formation of the complex in the HaCaT keratinocyte cell line (Figure 2c). Again, we observed a peak of interaction in the nucleus after 30 min UV-C irradiation, whereas no interaction was found between endogenous PIDD and PCNA in the cytoplasm. This probably means that the results shown in Figure $2 \mathrm{a}$, where constitutive interaction was found in the cytoplasm were most likely because of overexpression of PIDD (in accord with Figures $1 b$ and $d$ ). It is to note that the full-length form is well detected only using overexpression system (Figure 2a), as the endogenous one is really hard to detect. However, and importantly, only in the nucleus of this keratinocyte cell line, we could clearly detect the appearance and upregulation of the endogenous full-length PIDD form in response to UV-C irradiation (Figure 2c).

PIDD overexpression induces dissociation of p21 from PCNA. The interaction between PCNA and p21 is modulated during the UV-C response (see Introduction section): p21 detaches from PCNA after UV-C treatment (Supplementary Figure S2A, lane 6 versus 4) allowing PCNA to interact with the other partners involved in DNA repair. ${ }^{11}$ The results described above hinted at a possible role for PIDD in the process of p21 release from PCNA, as the peak of interaction between PIDD and PCNA was at 30 min post-UV-C closely coinciding with the start of p21 degradation (Figure 2a, lane 12). We therefore analyzed PCNA-p21 interaction, p21 degradation and PIDD upregulation in more detail, and found that all three events occur $\sim 30 \mathrm{~min}$ after UV-C treatment (Figure 3a, lanes 4-6). To mimic upregulation of PIDD expression seen upon UV-C exposure, we expressed increasing amounts of Flag-PIDD, which consequently led to PIDD binding correlating with p21 detachment from PCNA in a dose-dependent manner (Figure 3b). This suggests that at high-expression levels, PIDD is able to compete with p21 for PCNA binding. However, it is important to note that in this overexpression experiment, the levels of PIDD are probably higher than what would be found under physiological conditions. In addition, p21 is highly expressed in most cells and is known to have a strong affinity for PCNA. ${ }^{12}$ Therefore, it is possible that under physiological 
a

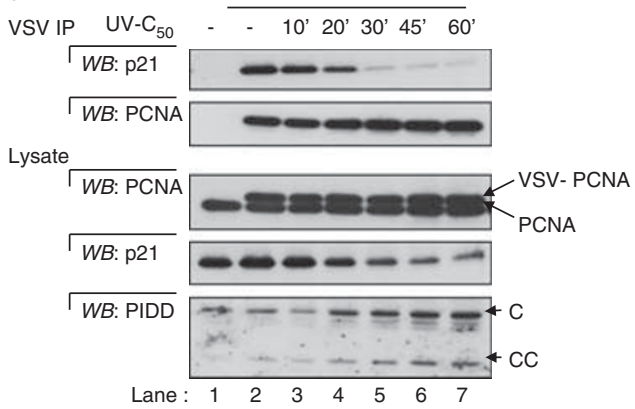

C

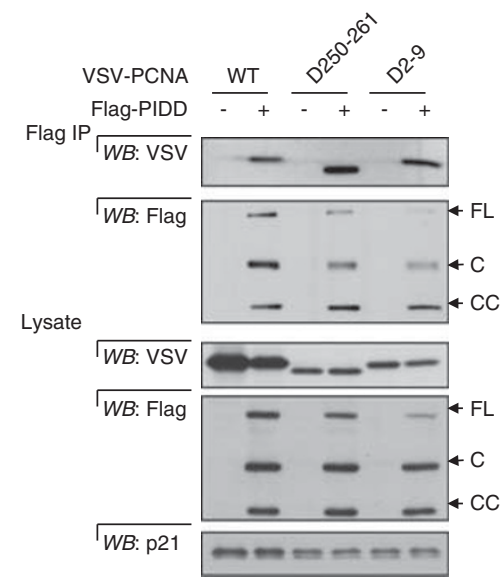

e

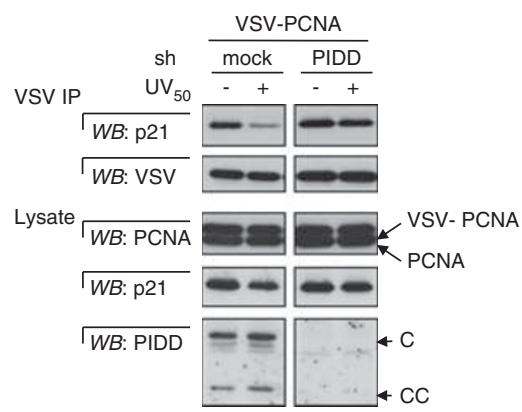

b VSV-PCNA - + + + + + PIDD-Flag - $\quad-0.10 .5 \quad 1 \quad 2$ VSV IP

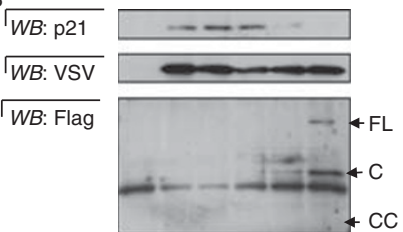

Lysate

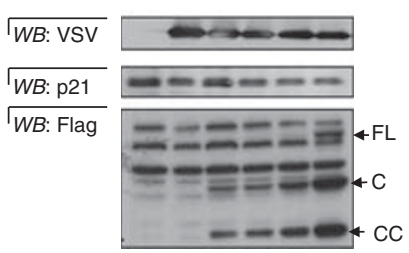

d
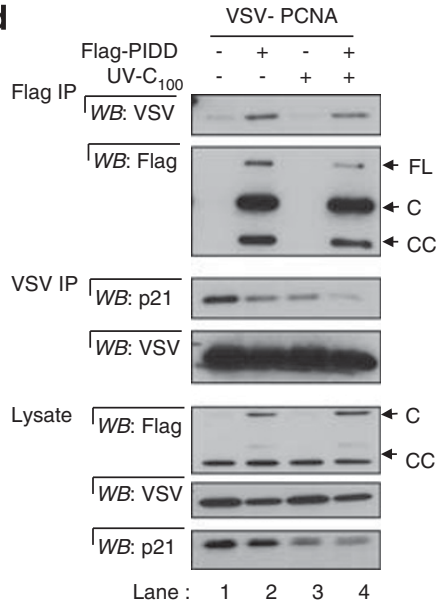

Figure 3 PIDD regulates the PCNA-p21 interaction in response to UV-C irradiation. (a-e) Where indicated, endogenous p21 interaction with PCNA was analysed by antiVSV IP, endogenous PCNA interaction with Flag-tagged PIDD was analysed after anti-Flag IP, and p21 degradation and PIDD expression were checked in total extracts. (a) PIDD upregulation correlates with PCNA-p21 detachment in the course of UV-C: 293T cells were transfected with VSV-tagged PCNA and subjected to different times of $50 \mathrm{~J} / \mathrm{m}^{2}$ UV-C irradiation. (b) PIDD overexpression is able to induce p21 detachment from PCNA: 293T cells were cotransfected with VSV-tagged PCNA and increasing amounts of Flag-tagged PIDD (the number indicates the ratio of Flag-PIDD plasmid transfected compared with the VSV-PCNA plasmid). (c) PCNA trimerization is not required for interaction with PIDD: 293T cells were cotransfected with differents constructs of VSV-tagged PCNA and Flag-tagged PIDD where indicated. See also Supplementary Figures S3a-c. (d) PIDD overexpression reduces p21-PCNA interaction in response to UV-C irradiation: 293T cells were transfected with VSV-tagged PCNA and with Flagtagged PIDD and subjected to $100 \mathrm{~J} / \mathrm{m}^{2} \mathrm{UV}$-C irradiation for $1 \mathrm{~h}$ where indicated. (e) PIDD deficiency leads to sustained p21-PCNA interaction in response to UV-C irradiation: 293 T cells stably knocked-down for PIDD or the corresponding control cells, were transfected with VSV-tagged PCNA and subjected to $50 \mathrm{~J} / \mathrm{m}^{2} \mathrm{UV}-\mathrm{C}$ irradiation for $30 \mathrm{~min}$ where indicated. All the figures are representative of at least three repetitions

conditions, PIDD upregulation amplifies rather than induces dissociation of $\mathrm{p} 21$ from PCNA upon UV-C irradiation. The dissociation of $\mathrm{p} 21$ from PCNA would suggest that PIDD and p21 interact with PCNA in close proximity and compete for nearby binding sites. We therefore used previously described PCNA deletion mutants $\left(\Delta 2-9\right.$ and $\left.\Delta 250-261^{12}\right)$ that are unable to form the trimeric PCNA structure and thereby prevent p21 binding (Supplementary Figure S3A). PIDD was still able to interact with these mutants (Figure $3 c$ ) indicating that the trimeric form of PCNA is not required for the interaction with PIDD. Another PCNA mutant (the KIE/A substitution mutant), ${ }^{12}$ capable of forming trimers, but unable to bind p21 (Supplementary Figure S3B), still interacts with PIDD (Supplementary Figure S3C), indicating that the p21 and PIDD binding sites are distinct. In addition, this competition is not because of direct binding of PIDD to p21 as no interaction was found between these two proteins (Supplementary Figure S2A). Hence, it is highly likely that the two binding partners of PCNA compete because of steric hindrance. 
a

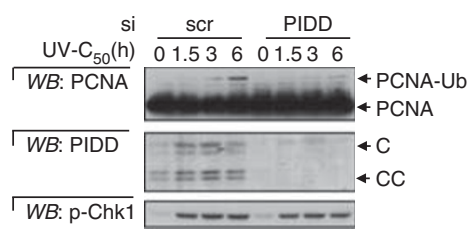

C

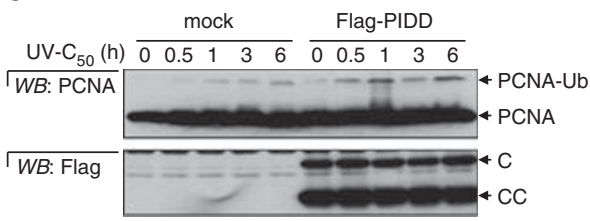

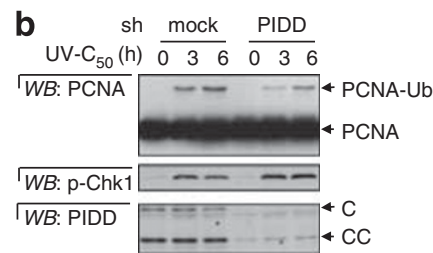

d sh mock PIDD 24h HU (mM) $\overline{02.55} \frac{\mathrm{m}}{02.55}$

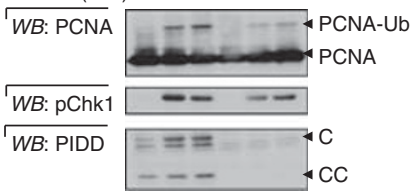

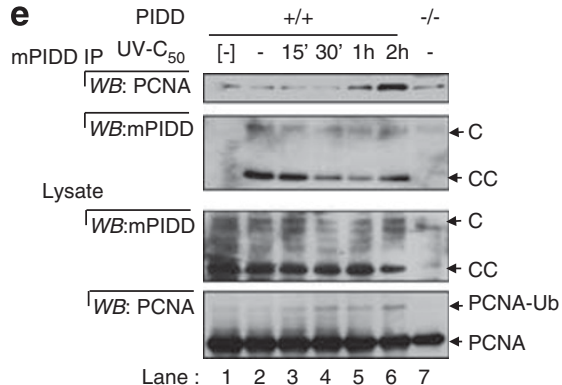

f

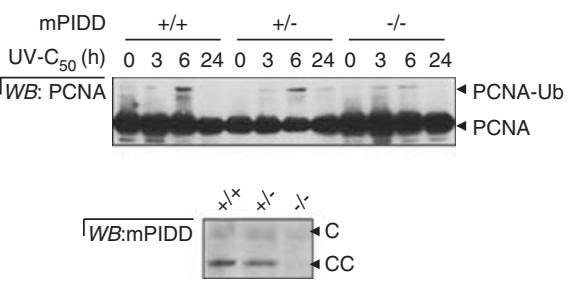

Figure 4 PIDD is involved in the monoubiquitination of PCNA in response to UV-C irradiation. (a-f) Cells were subjected to different times of $50 \mathrm{~J} / \mathrm{m}^{2} \mathrm{UV}-\mathrm{C}$ irradiation or $24 \mathrm{~h}$ to different doses of $\mathrm{HU}$ and monoubiquitination of PCNA was analyzed by western blotting as described in 'experimental procedures'. See also Figures S4b-c. (a) Hacat cells transfected with the indicated siRNAs (scr = scramble) or without siRNA as control. (b) HeLa cells stably knocked-down for PIDD or corresponding control cells. (c) The 293T cells stably overexpressing Flag-PIDD or corresponding control cells. (d) as in (b). (e) Primary keratinocytes from PIDD ${ }^{+/+}$mice were subjected to different times of $50 \mathrm{~J} / \mathrm{m}^{2} \mathrm{UV}-\mathrm{C}$ irradiation. Endogenous mPIDD was immunoprecipitated and endogenous PCNA interaction was analyzed $([-]=I P$ control was done with a control IgG antibody. Lane 7: keratinocytes from PIDD ${ }^{-I-}$ mice were also used as control). (f) Primary keratinocytes from PIDD ${ }^{+I+}$, PIDD $^{+I-}$ or PIDD ${ }^{-I-}$ mice (bottom panel show expression of mPIDD for each phenotype). All the figures are representative of at least two repetitions

As PIDD overexpression induces PCNA-p21 detachment (Figures $3 b$ and $d$, lane 2 versus 1 ), which is even more pronounced in response to UV-C (Figure 3d, lane 4 versus 3 ), we tested the PCNA-p21 interaction after UV-C irradiation in the absence of PIDD. In contrast to the overexpression experiments, we observed a sustained interaction between PCNA and p21 after short exposures to UV-C in cells where PIDD had been knocked down by shRNA (Figure $3 e$ ), confirming that PIDD has an active role in the destabilization of the PCNA-p21 interaction in response to UV-C.

PCNA-monoubiquitination in response to UV-C is PIDDdependent. In response to UV-C, PCNA is monoubiquitinated concomitant with p21 dissociation and degradation. ${ }^{9}$ As PIDD seems to act on the dissociation of p21 from PCNA, we investigated whether PIDD could promote PCNA monoubiquitination. Using both shRNA and siRNA strategies to reduce PIDD expression, monoubiquitination of PCNA in UV-irradiated cells was indeed strongly reduced (Figures $4 \mathrm{a}$ and b), while overexpression of PIDD enhanced PCNA monoubiquitination (Figure $4 \mathrm{c}$ ), confirming the important role of PIDD in this modification of PCNA. In contrast, phosphorylation and thus activation of Chk1, a checkpoint kinase activated during the UV response, was not affected (Figure 4a). PCNA is also monoubiquitinated in response to a variety of other DNA damaging agents, including methyl methanesulfonate (MMS), mitomycine $\mathrm{C}$, cisplatin and hydroxyurea $(\mathrm{HU}) .{ }^{23,25}$ PIDD deficiency also impaired PCNA monoubiquitination in response to these different agents, independently of the various cell lines used (Figure 4D, Supplementary Figures S4A and S4B).

PIDD interacts with RIP1 and with RAIDD/caspase-2 in a $\mathrm{DD}$-dependent manner, leading to the activation of $\mathrm{NF}-\kappa \mathrm{B}$ and caspase-2, respectively. To determine whether these established signaling pathways are implicated in PCNA monoubiquitination, we tested UV irradiation in cell lines knocked down for RAIDD and caspase-2, or overexpressing a dominant negative form of $\mathrm{l}_{\kappa} \mathrm{B} \alpha$, inhibiting the $\mathrm{NF} \kappa \mathrm{B}$ pathway. In none of these cells was UV-induced monoubiquitination of PCNA affected (Supplementary Figures S4C, S4D and S4E), indicating that neither the $\mathrm{NF}_{\kappa} \mathrm{B}$ pathway, nor the caspase-2PIDDosome activity was implicated. This is in agreement with our previous experiments indicating that the PIDD-PCNA complex does not rely on the PIDD-CC fragment. We took advantage of the PIDD $^{-1-}$ mice to confirm the results obtained with cell lines in which PIDD expression was impaired with siRNA. Cultures of mouse primary keratinocytes were generated and exposed to UV-C. We first confirmed the interaction between endogenous PIDD and PCNA in response to UV-C irradiation in these primary cells (Figure $4 \mathrm{e}$ ), and observed, in agreement with experiments obtained with cell lines, that keratinocytes of $\mathrm{PIDD}^{-/-}$mice 

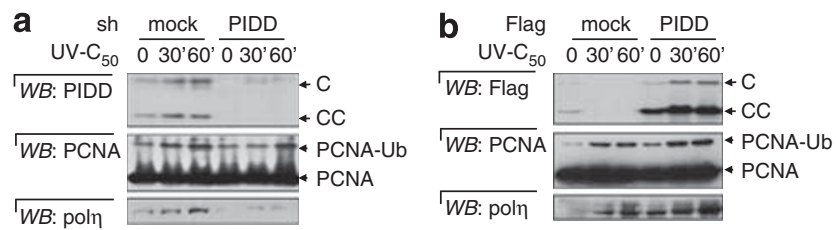

Figure 5 PIDD regulates the localization of pol $\eta$ to the chromatin in response to UV-C irradiation. (a and $\mathbf{b}$ ) Cells were subjected to different times of $50 \mathrm{~J} / \mathrm{m}^{2} \mathrm{UV}-\mathrm{C}$ irradiation and chromatin extraction was performed as described in 'experimental procedures'. Relocalization of PIDD, PCNA and pol $\eta$ to the chromatin was analyzed by western blot. (a) The 293T cells stably knocked-down for PIDD or corresponding control cells. (b) The 293T cells stably overexpressing Flag-PIDD or corresponding control cells. These two experiments were done two times and showed the same result

showed a defect in PCNA monoubiquitination (Figure 4f). The assembly of the PIDD-PCNA complex and the ubiquitination of PCNA both occurred later in these primary cells as compared with cell lines used before.

PIDD is required for pol $\eta$ localization to the chromatin in response to UV-C. Monoubiquitination of PCNA, which is the major modification of PCNA in response to UV-C, is associated with the recruitment of the polymerase pol $\eta$ to the chromatin to act on TLS. ${ }^{23}$ As PIDD affects PCNA monoubiquitination, we performed chromatin extraction on 293T cells after UV-C treatment and observed PCNA monoubiquitination, pol $\eta$ recruitment as well as PIDD recruitment to the chromatin, as expected (Figure 5a). Both PCNA monoubiquitination and pol $\eta$ recruitment were delayed in PIDD-deficient cells (Figure 5a), whereas both events were increased in response to UV-C when PIDD was overexpressed (Figure 5b).

PIDD deficiency is associated with increased apoptosis in response to UV-C. Efficient TLS is a crucial process in the prevention of cell death associated with stalled replication forks. Defects in TLS and pol $\eta$ recruitment, as well as prevention of PCNA monoubiquitination are associated with sensitization to apoptosis induced by UV-C irradiation. ${ }^{27,35}$ We thus investigated whether a defect in PCNA monoubiquitination and pol $\eta$ recruitment caused by PIDD deficiency sensitizes cells to UV-C induced apoptosis. Figure 6a shows that $\mathrm{HaCaT}$ cells with transiently knockeddown PIDD were more sensitive to apoptosis induced by UV-C (Figure 6). The same increase in cell death was observed in HeLa cells in which PIDD expression was reduced by shRNA (Supplementary Figure S5A).

To reproduce these results in a more physiological setting, we irradiated mice with UV-B to test the consequences of an impaired TLS in vivo. In agreement with our previous in vitro experiments, epidermis from irradiated $\mathrm{PIDD}^{-/-}$mice showed higher sensitivity to cell death induced by UV-B irradiation compared with WT as indicated by the higher number of cells containing active caspase-3 (Figures $6 c$ and $d$ and Supplementary figure S5B). The results indicate that PIDD deficiency participates in the sensitization of cells to apoptosis induced by UV irradiation in vivo.

\section{Discussion}

DNA lesions that are not repaired before $S$ phase are dangerous obstacles for the replication machinery, as most lesions cannot be accommodated into the active sites of the replicative DNA polymerases, thereby blocking progression of the replication fork. Prolonged stalling of replication forks can then lead to a collapse of the replication machinery, possibly resulting in double-strand breaks and gross chromosomal rearrangements, or even a permanent cell-cycle arrest with consequential cell death.

To steer clear of this potential crisis, cells have evolved the TLS bypass mechanism that handles stalled replication forks. One important feature of TLS is the switch from high fidelity replication polymerases to translesion polymerases that can incorporate either correct or incorrect nucleotides opposite the lesions demonstrating the disadvantage of the error prone TLS mechanism. Yet TLS probably exists to avoid even worse damage caused by stalled replication forks.

Bypass replication in response to DNA damage is directly controlled through covalent modifications of PCNA by monoubiquitin at residue Lys 164 . Our results show that at least one pathway leading to PCNA monoubiquitination and thus TLS requires the presence of PIDD. How PIDD influences PCNA ubiquitination, however, remains speculative. One possible scenario is that upon PIDD upregulation induced by UV-C, PIDD interacts strongly with PCNA thereby facilitates p21 detachment from PCNA. This may support the loading of PCNA to the DNA (probably with the RFC complex), its monoubiquitination by the RAD6-RAD18 complex and interaction with pol $\eta$ allowing TLS to be carried out.

P21 is well known as an important effector of cell cycle arrest after various genotoxic insults. P21 was also shown to modulate the monoubiquitination of PCNA and the interaction of PCNA with the TLS-specific polymerase $\eta .{ }^{27}$ We found that PIDD strongly binds to PCNA thereby impacting on the PCNAp21 interaction. Sustained binding of p21 to PCNA after UV-C treatment in the absence of PIDD correlates with a decrease of PCNA monoubiquitination and pol $\eta$ recruitment to the chromatin, in line with the inhibitory function of p21 in TLS. In keeping with this, inhibition of p21 degradation by the proteasome inhibitor MG132 completely inhibited PCNA monoubiquitination correlating with a sustained PCNA-p21 interaction (Supplementary Figures S6A and B), which confirms that dissociation and degradation of p21 is necessary for PCNA monoubiquitination., $9,25,36$

PIDD is a multifunctional protein, which is constitutively autoprocessed. Although the PIDD-C fragment triggers the RIP1-mediated NF- $\kappa$ B pathway, PIDD-CC sensitizes cells to apoptosis through recruitment of RAIDD/caspase- $2 .{ }^{30} \mathrm{~A}$ third function is mediated by the PIDD-DNA-PKcs-caspase-2 complex that is implicated in the maintenance of a G2/M DNA checkpoint and DNA repair by the NHEJ pathway. ${ }^{32}$ The involvement of PIDD in TLS is a fourth function that appears to act independently of the three other complexes. Neither caspase-2, RAIDD deficiency nor $\mathrm{NF} \kappa \mathrm{B}$ inhibition influences PIDD-induced PCNA monoubiquitination. This 'independence' is in agreement with the fact that, in contrast to the DD-dependent interaction with RIP1, RAIDD and DNA-PKcs, it is the $\mathrm{N}$-terminal part and most probably the Zu5 domains of 
a

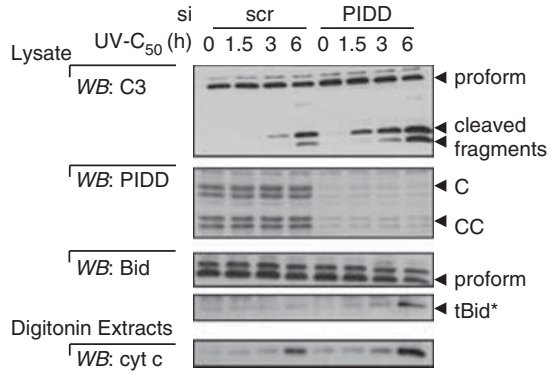

b

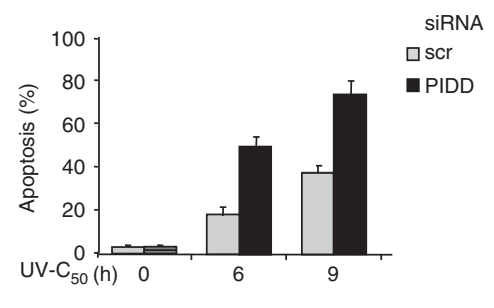

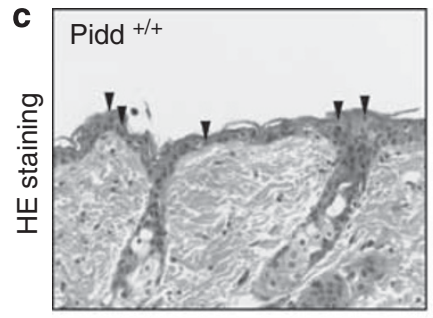

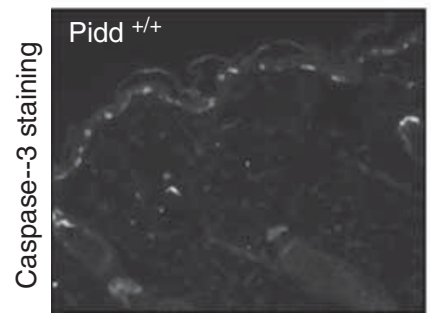

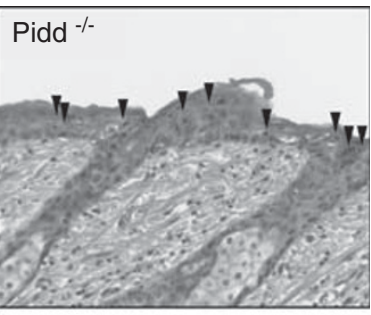

\section{Pidd}

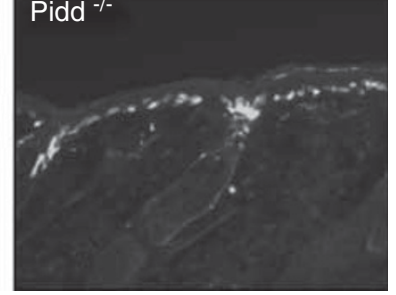

d
$P=0.0125$

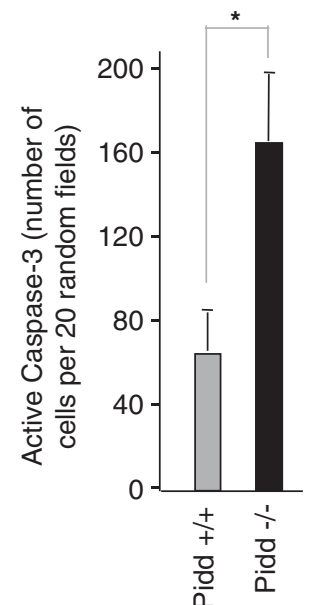

UV-B $200 \mathrm{~mJ} / \mathrm{cm}^{2}$

Figure 6 PIDD deficiency sensitizes cells to UV-C induced apoptosis. (a and $\mathbf{b})$ Hacat cells were transfected with the indicated siRNA and subjected to different times of $50 \mathrm{~J} / \mathrm{m}^{2} \mathrm{UV}$-C irradiation. (a) Markers of apoptosis, Bid and caspase-3 cleavage as well as Cytochrome $c$ release were analysed by western-blotting. See also Supplementary Figure S5A. (b) Apoptosis was quantified by Hoechst staining and show the mean of two independent experiments. (c and d) PIDD ${ }^{+1+}$ or ${ }^{-l-}$ mice were irradiated as described in 'experimental procedures'. Apoptosis was detected by HE staining (arrowheads indicate sunburn cells) and active caspase-3 staining (c) and quantification (d). See also Supplementary Figure S5b. Data shown are representative of at least three independent experiments and reported as mean \pm S.D. $P$-value for statistical significance was calculated using the Student's $t$-test and considered significant $\left(^{*}\right)$

PIDD that are responsible for its interaction with PCNA and RFC proteins. It is currently not clear if PIDD-FL, the PIDD-C form or both are responsible for the interaction, in part because the endogenous full-length form is hardly detectable by western blotting.

We identified in PIDD a sequence that could resemble a PIP-domain located in the first Zu5 domain in position aa 391 and that is conserved in mice: ' $x A x Q x x V x x W$ '. We mutated the core sequence ( $Q x x V$ converted in $A x x A)$ and tested the interaction of PCNA with this mutant. Surprisingly, the mutation of this sequence mostly affects the processing of PIDD, shown by the accumulation of the full-length isoform (Supplementary Figure S7, lane 3 versus 2 in total extract).

For cells, it may often be preferable to risk the introduction of point mutations during TLS over other mechanisms of DNA repair, which can cause chromosomal aberrations or widespread cell death. In cell lines lacking expression of PIDD and thus functional TLS, we indeed found increased cell death in accordance with published data. ${ }^{27}$ This cell death is characterized by caspase- 3 activation, but the nature of the upstream caspase(s) leading to caspase-3 processing is currently unclear. Caspase-2 was shown to be involved in DNA damage-induced cell death. As cell death can occur in the absence of PIDD, the PIDD-RAIDD-caspase-2 and the
PIDD-DNA-PKcs-caspase-2 complexes are unlikely to be implicated. Yet, it has recently been found that caspase-2 activation can also occur independently of the PIDDosome. ${ }^{37}$ It remains to be shown therefore whether caspase-2 is implicated in UV-induced cell death. At least in vivo, skin of PIDD-/- mice exposed to UV irradiation showed an increase of apoptotic cells as compared with wild-type cells.

In summary, our results demonstrate an important role of PIDD in the regulation of PCNA during the DNA damage response induced by UV irradiation, controlling TLS and apoptosis.

\section{Materials and Methods}

Cell culture and UV irradiation. Human embryonic kidney 293T cells, and human HeLa cervical carcinoma cells were grown in DMEM + Glutamax (Life Technologies, Basel, Switzerland), supplemented with $10 \%$ fetal calf serum and $100 \mathrm{U} / \mathrm{ml}$ penicillin, $100 \mu \mathrm{g} / \mathrm{ml}$ streptomycin. The generation of stably expressing PIDD cells was achieved by retroviral infection as described previously, and cells stably knocked down for PIDD were obtained by lentiviral infection using shRNA from Openbiosystems (Hunstville, TX, USA) as described elsewhere. ${ }^{29,38}$ UV-C irradiation were performed with a Stratalinker UV crosslinker 2400 (Stratagene, Agilent Technologies, Basel, Switzerland) equipped with $254 \mathrm{~nm}$ tubes. Medium was removed from cells before irradiation and replenished after irradiation.

Transient transfection. Transient transfection of both expression vectors or siRNA were performed using the DNA-calcium phosphate precipitation method and 
incubated for $48 \mathrm{~h}$ before treatment and harvesting of the cells. The total amount of DNA was kept constant by adding empty vector where necessary.

Coimmunoprecipitation and western blotting. Immunoprecipitation (IP) or colP experiments with transfected proteins were performed in lysis buffer containing 1\% NP-40, $20 \mathrm{mM}$ Tris, pH 7.4, $250 \mathrm{mM} \mathrm{NaCl}, 1 \mathrm{mM}$ EDTA, $5 \%$ glycerol and a protease inhibitor cocktail. After lysis, the extracts were incubated with antiFlag or anti-VSV antibody for $4 \mathrm{~h}$ to overnight. After incubation the beads were washed three times with lysis buffer, and analyzed by SDS-PAGE and immunoblotting.

For PCNA analysis, non extractable (NE) fractions were obtained with Triton X-100. Briefly, cells were lysed $1 \mathrm{~min}$ on ice in PBS Triton X-100 $1 \%$ before centrifugation $(10 \mathrm{~min}, 10000 \mathrm{~g})$. Supernatants were removed and pellets resuspended in sample buffer and boiled before western blotting.

For Cytochrome $c$ release analysis, cytoplasmic extraction was performed with Digitonine (Sigma-Aldrich, Buchs, Switzerland). Briefly, cells were lysed in Digitonine lysis buffer $\left(75 \mathrm{mM} \mathrm{NaCl}, 1 \mathrm{mM} \mathrm{NaH} \mathrm{PO}_{4}, 8 \mathrm{mM} \mathrm{Na}_{2} \mathrm{HPO}_{4}, 250 \mathrm{mM}\right.$ sucrose, $100 \mu \mathrm{g} / \mathrm{ml}$ Digitonine) and incubated $10 \mathrm{~min}$ on ice before centrifugation.

The antibodies used for western blotting were: anti-VSV and anti-Flag (Sigma-Aldrich), anti-p21 (C-19) and anti-PCNA (PC10) (Santa Cruz, Nuningen, Switzerland), anti-mouse PIDD (Lise-1) (Adipogen, Epalinges, Switzerland), antiPIDD (Anto-1) and anti-Cytochrome $c$ (Axxora, Lausen, Switzerland), anti-RFC5 and anti-phospho-Chk1 (S317) (Bethyl, Lucerne, Switzerland), anti-DNA polymerase eta and anti-lamin B1 (Abcam, Cambridge, UK), anti-caspase-3 (BD, Allschwil, Switzerland) and anti-Bid (R\&D sytems, Abingdon, UK).

Chromatin extraction. For chromatin fractionation experiments, cells were lysed $5 \mathrm{~min}$ on ice in solution $\mathrm{A}$ ( $10 \mathrm{mM}$ HEPES $\mathrm{pH} 7.9,10 \mathrm{mM} \mathrm{KCl}, 1.5 \mathrm{mM} \mathrm{MgCl}_{2}$, $0.34 \mathrm{M}$ sucrose, $10 \%$ glycerol, $1 \mathrm{mM}$ DTT, $10 \mathrm{mM} \mathrm{NaF}, 1 \mathrm{mM} \mathrm{Na}_{2} \mathrm{VO}_{3}$, proteases inhibitors $+0.1 \%$ Triton $\mathrm{X}-100$ added just before use). Nuclei were separated by centrifugation ( $4 \mathrm{~min}, 1300 \times \mathrm{g}$ ), washed once in solution $\mathrm{A}$ (without Triton) and then lysed $30 \mathrm{~min}$ on ice in solution B ( $3 \mathrm{mM}$ EDTA, $0.2 \mathrm{mM}$ EGTA, $1 \mathrm{mM}$ DTT, proteases inhibitors). Insoluble chromatin was then separated by centrifugation $(5 \mathrm{~min}, 1700 \times \mathrm{g})$, washed once in solution $\mathrm{B}$, collected by centrifugation $(1 \mathrm{~min}$, $10000 \times g$ ), resuspended in sample buffer and sonicated before immunoblotting.

Cytoplasm/nucleus fractionation. Cytoplasm/nucleus fractionation experiments are based on a previous report. ${ }^{39}$ Briefly, cells were harvested and washed once in ice-cold PBS. Pellets were resuspended in $2 \times$ volume of buffer $\mathrm{C}$ (10 mM HEPES pH 7.9, $10 \mathrm{mM} \mathrm{KCl}, 1.5 \mathrm{mM} \mathrm{MgCl}_{2}, 300 \mathrm{mM}$ sucrose, $0.5 \% \mathrm{NP} 40$, proteases inhibitors $+0.5 \mathrm{mM}$ DTT added at the last minute) and left $5 \mathrm{~min}$ on ice. Nuclei and cytosolic fractions were separated by centrifugation ( 45 s, 9000 r.p.m., $4^{\circ} \mathrm{C}$ ). Nuclei were washed once with buffer $\mathrm{C}$, resuspended in $2 \times$ volume of buffer $\mathrm{N}(20 \mathrm{mM}$ HEPES pH 7.9, $100 \mathrm{mM} \mathrm{NaCl}, 0.2 \mathrm{mM}$ EDTA, 20\% glycerol, $100 \mathrm{mM} \mathrm{KCl}$, proteases inhibitors $+0.5 \mathrm{mM}$ DTT added just before use) and then subjected to three cycles of freeze (liquid $\mathrm{N}_{2}$ ) and thaw $\left(37^{\circ} \mathrm{C}\right.$ ) before sonication. Nuclear fractions were then isolated by centrifugation (10 min, 13000 r.p.m.). In westernblots, caspase-3 and lamin-B1 were used as controls of fraction purity.

Apoptosis quantification. Apoptosis was determined by Hoechst 33342 $\left(1 \mu \mathrm{g} / \mathrm{ml}\right.$, Sigma-Aldrich) staining of the cells for $30 \mathrm{~min}$ at $37^{\circ} \mathrm{C}$, and fluorescence microscopy analysis of 200 cells per condition.

Primary keratinocytes extraction. Adult mice (8-12 weeks old) were killed and the tails removed. The tail skin was peeled off and cut into $1 \mathrm{~cm}$ fragments, which were incubated overnight at $4{ }^{\circ} \mathrm{C}$ in Dipase II solution $(8 \mathrm{mg} / \mathrm{ml} \mathrm{in}$ PBS, Roche, Basel, switzerland). The following day, the epidermis was separated, incubated for $5 \mathrm{~min}$ at $37^{\circ} \mathrm{C}$ in Trypsin/EDTA 0,05\% (Invitrogen, Basel, Switzerland), and filtered through a $70 \mu \mathrm{m}$ cell strainer. Cells were seeded on collagen IV-coated plates (BD) at the density of 200000 cells $/ \mathrm{ml}$, in Epilife medium (Biocoba, Reinach, Switzerland) supplemented with HGKS (Biocoba) for 4 days before UV irradiation.

Immunohistochemistry. The irradiated skin was processed by paraffin embedding and sections were stained by hematoxylin-eosin ( $\mathrm{HE})$, or using an antiactive caspase-3 antibody. Briefly, after hydration, paraffin sections were heated three times for $5 \mathrm{~min}$ in Citrate $0.01 \mathrm{M}$ buffered at $\mathrm{pH} 6.0$ using a microwave at $620 \mathrm{~W}$ and cooled on ice. PBS containing $0.2 \%$ Triton X-100 and $10 \%$ normal goat serum was used for blocking. The sections were then stained using anti-active caspase-3 antibody (AF835, R\&D Systems) overnight at $4{ }^{\circ} \mathrm{C}$ at $0.5 \mathrm{ng} / \mathrm{ml}$ and revealed with an Alexa488-coupled goat anti-rabbit antibody (A11034, Life Technologies) used at $10 \mu \mathrm{g} / \mathrm{ml}$ in PBS $0.2 \%$ Triton X-100, then counterstained with DAPI for nuclear staining (Roche Diagnostics), as described in the manufacturer's instructions. The slides were mounted in FluorSave Reagent (Merck, Nottingham, UK) and images were acquired using a fluorescent microscope (Zeiss Axiophot, Zeiss AG, Feldbach, Switzerland).

Identification of PIDD-binding partners by mass-spectrometry Cytoplasmic and nuclear fractionations. Around $10^{6} 293 \mathrm{~T}$ cells stably expressing Flag-PIDD or control cells (obtained by retroviral infection as described in experimental procedures) were harvested and washed two times with PBS and then two times with PBS $10 \mathrm{mM}$ sodium butyrate. Cells were lysed in $2 \mathrm{ml}$ lysis buffer containing $0.1 \%$ Triton $\mathrm{X}-100,250 \mathrm{mM}$ sucrose, $10 \mathrm{mM}$ Tris $\mathrm{pH} 7.4,10 \mathrm{mM}$ sodium butyrate, $4 \mathrm{mM} \mathrm{MgCl}_{2}$ and complete protease inhibitor (PI, Roche). Cells were gently disrupted in a loose Dounce homogenizer ( 25 strokes) and the resulting lysate was centrifugated at 4500 r.p.m. at $4{ }^{\circ} \mathrm{C}, 10 \mathrm{~min}$. The supernatant corresponding to the cytoplasmic fraction was cleared by centrifugation at 13000 r.p.m. at $4{ }^{\circ} \mathrm{C}, 10 \mathrm{~min}$. The pellet was resuspended in $4 \mathrm{ml}$ of buffer $\mathrm{C}$ containing $250 \mathrm{mM}$ sucrose, $10 \mathrm{mM}$ Tris $\mathrm{pH} 7.4,10 \mathrm{mM}$ sodium butyrate, $4 \mathrm{mM}$ $\mathrm{MgCl}_{2}$ and $\mathrm{PI}$, put on a $30 \%$ sucrose cushion (30\% sucrose in buffer $\mathrm{C}$ ) and centrifuged at 3300 r.p.m., $5 \mathrm{~min}$ at $4^{\circ} \mathrm{C}$ (swing-out rotor, brake set to minimum). The pellet containing the nuclei was resuspended in $1 \mathrm{ml}$ buffer $\mathrm{N}$ containing $0.5 \%$ NP-40, $10 \%$ glycerol, $420 \mathrm{mM} \mathrm{NaCl}, 20 \mathrm{mM}$ Hepes pH 7.9, $1.5 \mathrm{mM} \mathrm{MgCl}, 1 \mathrm{mM}$ DTT and PI. Nuclear extracts were incubated on a rotating wheel at $4{ }^{\circ} \mathrm{C}$ for $30 \mathrm{~min}$, homogenized in a loose Dounce homogenizer (20 strokes) and clarified by centrifugation at 13000 r.p.m. $4^{\circ} \mathrm{C}$ for $20 \mathrm{~min}$. Nuclear fractions were diluted before immunoprecipitations with buffer $C$ without salt to obtain a $150 \mathrm{mM} \mathrm{NaCl}$ concentration. Tris $\mathrm{pH} 7.4$ and $\mathrm{NaCl}$ were added to cytoplasmic fractions to obtain a $50 \mathrm{mM}$ and $150 \mathrm{mM}$ concentration, respectively, before immunoprecipitations (described in Experimental procedures).

Fractionation, digestion and analysis by mass spectrometry. Eluted proteins from IP were separated on a $10 \%$ mini SDSpolyacrylamide gel and stained with Coomassie blue. Entire gel lanes were excised into 20 equal regions from top to bottom and digested with trypsin (Promega, Dübendorf, Switzerland) as described (1,2). Data-dependent LC-MS/MS analysis of extracted peptide mixtures after digestion was carried out on a hybrid linear trap LTQ-Orbitrap mass spectrometer (Thermo Fisher Scientific, Huntsville, TX, USA) interfaced to an Agilent 1100 nanocapillary HPLC equipped with a $75 \mu \mathrm{m}$ internal diameter $\mathrm{C} 18$ reversed-phase column. Collections of tandem mass spectra for database searching were generated from raw data and searched using Mascot (Matrix Science, London, UK; version 2.1.0) against the release 11.0 of the UNIPROT database, (SWISSPROT + TrEMBL, http://www.expasy.org) restricted to human taxonomy. The software Scaffold (version Scaffold-01_06_03, Proteome Software Inc., Portland, OR, USA) was used to validate MS/MS based peptide (minimum $95 \%$ probability (4)) and protein ( $\min 99 \%$ probability (3)) identifications, perform dataset alignment and subtraction as well as parsimony analysis to discriminate homologous hits. In an additional filtering step, only proteins identified with at least three spectra were retained for further analysis.

\section{Conflict of interest}

The authors declare no conflict of interest.

Acknowledgements. We thank $\mathrm{N}$ Aebi, S Roques and $\mathrm{A}$ Yazdi for technical support, all the members of the A Constantinou's Lab and P Schneider for helpful discussions, and Catherine Dostert for critical reading of the manuscript. This work was supported by grants from Hermione, Apo-SYS and ApopTrain European Projects EL and MJE are supported by FEBS fellowships.

\section{Author contributions}

EL, SMS, AV, OG and JT designed the research, analysed the data and wrote the paper; EL, SMS, MJE, LXH, BJ, CM, AT and MQ performed the research.

1. Moldovan GL, Pfander B, Jentsch S. PCNA, the maestro of the replication fork. Cell 2007; 129: $665-679$. 
2. Maga G, Hubscher U. Proliferating cell nuclear antigen (PCNA): a dancer with many partners. J Cell Sci 2003; 116 (Part 15): 3051-3060

3. Warbrick E. The puzzle of PCNA's many partners. Bioessays 2000; 22: 997-1006.

4. Majka J, Burgers PM. The PCNA-RFC families of DNA clamps and clamp loaders. Prog Nucleic Acid Res Mol Biol 2004; 78: 227-260.

5. Johnson A, Yao NY, Bowman GD, Kuriyan J, O'Donnell M. The replication factor C clamp loader requires arginine finger sensors to drive DNA binding and proliferating cell nuclear antigen loading. J Biol Chem 2006; 281: 35531-35543.

6. Majka J, Chung BY, Burgers PM. Requirement for ATP by the DNA damage checkpoint clamp loader. J Biol Chem 2004; 279: 20921-20926.

7. Podust VN, Podust LM, Goubin F, Ducommun B, Hübscher U. Mechanism of inhibition of proliferating cell nuclear antigen-dependent DNA synthesis by the cyclin-dependent kinase inhibitor p21. Biochemistry 1995; 34: 8869-8875.

8. Bruning JB, Shamoo Y. Structural and thermodynamic analysis of human PCNA with peptides derived from DNA polymerase-delta p66 subunit and flap endonuclease-1. Structure 2004; 12: 2209-2219.

9. Bendjennat M, Boulaire J, Jascur T, Brickner H, Barbier V, Sarasin A et al. UV irradiation triggers ubiquitin-dependent degradation of $\mathrm{p} 21$ (WAF1) to promote DNA repair. Cell 2003; 114: 599-610.

10. Shivji MK, Grey SJ, Strausfeld UP, Wood RD, Blow JJ. Cip1 inhibits DNA replication but not PCNA-dependent nucleotide excision-repair. Curr Biol 1994; 4: 1062-1068.

11. Waga S, Hannon GJ, Beach D, Stillman B. The p21 inhibitor of cyclin-dependent kinases controls DNA replication by interaction with PCNA. Nature 1994; 369: 574-578.

12. Oku T, Ikeda S, Sasaki H, Fukuda K, Morioka H, Ohtsuka E et al. Functional sites of human PCNA which interact with p21 (Cip1/Waf1), DNA polymerase delta and replication factor C. Genes Cells 1998; 3: 357-369.

13. Gary R, Ludwig DL, Cornelius HL, Macinnes MA, Park MS. The DNA repair endonuclease XPG binds to proliferating cell nuclear antigen (PCNA) and shares sequence elements with the PCNA-binding regions of FEN-1 and cyclin-dependent kinase inhibitor p21. J Biol Chem 1997; 272: 24522-24529.

14. Nichols AF, Sancar A. Purification of PCNA as a nucleotide excision repair protein. Nucleic Acids Res 1992; 20: 2441-2446.

15. Shivji KK, Kenny MK, Wood RD. Proliferating cell nuclear antigen is required for DNA excision repair. Cell 1992; 69: 367-374.

16. Friedberg EC. Suffering in silence: the tolerance of DNA damage. Nat Rev Mol Cell Biol 2005; 6: 943-953.

17. Lehmann E, Brueckner F, Cramer P. Molecular basis of RNA-dependent RNA polymerase II activity. Nature 2007; 450: 445-449.

18. McCulloch SD, Kokoska RJ, Masutani C, Iwai S, Hanaoka F, Kunkel TA. Preferential cissyn thymine dimer bypass by DNA polymerase eta occurs with biased fidelity. Nature 2004; 428: $97-100$

19. Washington MT, Johnson RE, Prakash L, Prakash S. Accuracy of lesion bypass by yeast and human DNA polymerase eta. Proc Natl Acad Sci USA 2001; 98: 8355-8360.

20. Kannouche PL, Lehmann AR. Ubiquitination of PCNA and the polymerase switch in human cells. Cell Cycle 2004; 3: 1011-1013.

21. Hoege C, Pfander B, Moldovan GL, Pyrowolakis G, Jentsch S. RAD6-dependen DNA repair is linked to modification of PCNA by ubiquitin and SUMO. Nature 2002; 419 135-141.
22. Watanabe K, Tateishi S, Kawasuji M, Tsurimoto T, Inoue H, Yamaizumi M. Rad18 guides poleta to replication stalling sites through physical interaction and PCNA monoubiquitination. EMBO J 2004; 23: 3886-3896.

23. Kannouche PL, Wing J, Lehmann AR. Interaction of human DNA polymerase eta with monoubiquitinated PCNA: a possible mechanism for the polymerase switch in response to DNA damage. Mol Cell 2004; 14: 491-500.

24. Bienko M, Green CM, Crosetto N, Rudolf F, Zapart G, Coull B et al. Ubiquitin-binding domains in $Y$-family polymerases regulate translesion synthesis. Science 2005; 310: 1821-1824.

25. Soria G, Podhajcer O, Prives C, Gottifredi V. P21Cip1/WAF1 downregulation is required for efficient PCNA ubiquitination after UV irradiation. Oncogene 2006; 25: 2829-2838.

26. Avkin S, Sevilya Z, Toube L, Geacintov N, Chaney SG, Oren M et al. p53 and p21 regulate error-prone DNA repair to yield a lower mutation load. Mol Cell 2006; 22: 407-413.

27. Soria G, Speroni J, Podhajcer OL, Prives C, Gottifredi V. p21 differentially regulates DNA replication and DNA-repair-associated processes after UV irradiation. J Cell Sci 2008; 121 (Part 19): 3271-3282.

28. Lin Y, Ma W, Benchimol S. Pidd, a new death-domain-containing protein, is induced by p53 and promotes apoptosis. Nat Genet 2000; 26: 122-127.

29. Tinel A, Tschopp J. The PIDDosome, a protein complex implicated in activation of caspase2 in response to genotoxic stress. Science 2004; 304: 843-846.

30. Tinel A, Janssens S, Lippens S, Cuenin S, Logette E, Jaccard B et al. Autoproteolysis of PIDD marks the bifurcation between pro-death caspase-2 and pro-survival NF-kappaB pathway. EMBO J 2007; 26: 197-208

31. Janssens S, Tinel A, Lippens S, Tschopp J. PIDD mediates NF-kappaB activation in response to DNA damage. Cell 2005; 123: 1079-1092.

32. Shi M, Vivian CJ, Lee KJ, Ge C, Morotomi-Yano K, Manzl C et al. DNA-PKcs-PIDDosome: a nuclear caspase-2-activating complex with role in $\mathrm{G} 2 / \mathrm{M}$ checkpoint maintenance. Cell 2009; 136: 508-520.

33. Balajee AS, May A, Dianova I, Bohr VA. Efficient PCNA complex formation is dependent upon both transcription coupled repair and genome overall repair. Mutat Res 1998; 409: 135-146.

34. Prosperi E, Stivala LA, Sala E, Scovassi Al, Bianchi L. Proliferating cell nuclear antigen complex formation induced by ultraviolet irradiation in human quiescent fibroblasts as detected by immunostaining and flow cytometry. Exp Cell Res 1993; 205: 320-325.

35. Brown S, Niimi A, Lehmann AR. Ubiquitination and deubiquitination of PCNA in response to stalling of the replication fork. Cell Cycle 2009; 8: 689-692.

36. Livneh Z. Keeping mammalian mutation load in check: regulation of the activity of errorprone DNA polymerases by p53 and p21. Cell Cycle 2006; 5: 1918-1922.

37. Manzl C, Krumschnabel G, Bock F, Sohm B, Labi V, Baumgartner F et al. Caspase-2 activation in the absence of PIDDosome formation. J Cell Biol 2009; 185: 291-303.

38. Papin S, Cuenin S, Agostini L, Martinon F, Werner S, Beer HD et al. The SPRY domain of Pyrin, mutated in familial Mediterranean fever patients, interacts with inflammasome components and inhibits prolL-1beta processing. Cell Death Differ 2007; 14: $1457-1466$.

39. Dignam JD, Lebovitz RM, Roeder RG. Accurate transcription initiation by RNA polymerase II in a soluble extract from isolated mammalian nuclei. Nucleic Acids Res 1983; 11 : $1475-1489$.

Supplementary Information accompanies the paper on Cell Death and Differentiation website (http://www.nature.com/cdd) 